\title{
2-Hop Neighbors Table Based Broadcasting Algorithm in Ad Hoc Network
}

\author{
Leqiang Bai \\ Information \& Control Engineering Faculty \\ Shenyang Jianzhu University \\ Shenyang, China \\ e-mail: baileqiang@sjzu.edu.cn
}

Ye Tian

Information \& Control Engineering Faculty

Shenyang Jianzhu University

Shenyang, China

e-mail: t3jds@126.com

\author{
Jing Dai \\ Information \& Control Engineering Faculty \\ Shenyang Jianzhu University \\ Shenyang, China \\ e-mail: daijing6615@163.com \\ Jingjing Sun \\ Information \& Control Engineering Faculty \\ Shenyang Jianzhu University \\ Shenyang, China \\ e-mail: sjjwang402@163.com
}

\begin{abstract}
In order to reduce redundant forwarding nodes and transmission delay, this paper proposes a 2-hop neighbors table based broadcasting algorithm. The proposed algorithm introduces two conceptions which are in-degree of two hop neighbor nodes and out-degree of neighbor nodes. It can reduce the number of forwarding nodes and redundant forwarding that using the out-degree and in-degree of nodes chooses the forwarding nodes from neighbor nodes. The simulation results show that the proposed algorithm has lower ratio of forwarding and smaller transmission delay, the performance is good.
\end{abstract}

Keywords-ad hoc network; broadcasting algorithm; two hop neighbors table; forwarding nodes

\section{INTRODUCTION}

Wireless ad hoc network is a special network without relying on any architecture to support. Compared with the traditional wireless network, wireless ad hoc network has the characteristics of no center, self-organization. There is a large number of application prospects in the military, industrial control, etc. Broadcasting is a very significant operation in wireless ad hoc network. Broadcasting is one of the most fundamental operations in Ad Hoc networks. Most of the major routing protocols, such as DSR, AODV, ZRP, LAR, etc., rely on broadcasting for disseminating route discovery, route maintenance, or topology update packets. Broadcasting is a very frequently invoked utility function in $\mathrm{Ad} \mathrm{Hoc} \mathrm{networks.} \mathrm{So} \mathrm{many} \mathrm{scholars} \mathrm{pay}$ attention to the broadcasting algorithm, they have also got big achievements in recent years.

The most simple and basic broadcasting operation is flooding which every node transmits the message after receiving it for the first time. However flooding can impose a large number of redundant transmissions, which can result in channel conflict, network congestion and broadcast storm. In order to solve the problems of flooding, many new broadcasting algorithms have been proposed in recent years. Existing broadcasting algorithms have been classified into five types in [1]: probabilistic scheme, counter-based scheme, distance-based scheme, location- based scheme, cluster-based scheme. Basic idea of probabilistic scheme is to use a predefined probability to decide whether to forward [2].Basic idea of counter-based scheme is to use a predefined counter to decide whether to forward [3]. The two algorithms do not need neighbor nodes information, but due to the use of predefined thresholds their performance will get bad when the node density increase. Basic ideas of distance-based scheme and location-based scheme are both that if the nodes could cover more additional area, the nodes will be the forwarding nodes. The two methods do not need to record accepting state and do not need the information of neighbor nodes, so the transmission delay and Storage cost are both very small. But due to the use of predefined thresholds the performance will get bad when node density or network load change. And the distribution of the forwarding nodes gets unreasonable because the forwarding nodes don't know the relationship of location each other. It will loss packets when some non redundant nodes make mistakes, and it will even stop forwarding.

\section{RELATED WORKS}

The author of [4] proposes a broadcasting algorithm based on neighbor nodes information, which can reduce ratio of forwarding greatly under the premise in guarantee ratio of delivery. And it does not need location information supported by GPS technology. The author of [5] classifies existing broadcasting algorithms based on neighbor nodes information into active and passive. The author of [6] divides the broadcasting algorithms based on neighbor nodes information into dynamic and static. Broadcasting using the static approach is a NP problem without location information. The author of [7] proposes an efficient flooding scheme that uses only 1-hop neighbor information, it have been proved the proposed scheme achieves the local optimality in terms of: 1) the number of forwarding nodes is the minimal and 2) the time complexity is the lowest. The author of [8] proposes an efficient and reliable broadcast protocol for clustered WSN (ERBP). In ERBP, nodes need its 1-hop neighborhood information so that the 
cluster head could establish a small route table used to transmit message to neighbor cluster head.

The broadcasting algorithm based on neighbor nodes information both send the "Hello" packets to collect the information of neighbor nodes and two hop neighbor nodes, and control node whether to forward the packets with local topology information. The existing broadcasting algorithms based on neighbor node information are divided into three kinds mainly: neighbor-designating, self-pruning and cluster broadcasting algorithm. Neighbor-designating broadcasting algorithm can restrain redundant forwarding however the effect is not obvious, it cannot adapt to the dynamic network which change frequently [9]. Considering of node connectivity, remaining energy and node relative distance, the author of paper [10] advances a new neighbor-designating broadcasting algorithm that is weighted-based symmetrical area broadcasting algorithm. According to the node weights, it constructs and determines independent dominating sets, and selects candidate control node connect to independent dominating sets. And it optimizes the broadcast algorithm, eliminates some redundant forwarding nodes and enhances the performance of the broadcast algorithm. Self-pruning broadcasting algorithm's adaptability is strong, it can adapt to the network which topology changes frequently [11]. Each broadcasting packet's header will become very big when the number of nodes is large, this can lead to packet data section is greatly compressed. Cluster broadcasting algorithm could reduce the redundant forwarding nodes greatly in the dense network, however its ratio of forwarding is not good [12]. To solve the problem that the ratio of forwarding broadcasting algorithm based on neighbor nodes information is on the high side in sparse network, this paper proposed 2-hop neighbors table based broadcasting algorithm (2NTB).

\section{2NTB ALGORITHM}

\section{A. Algorithm Parameters and Definitions}

This paper defines three types of nodes: neighbor nodes, two hop neighbor nodes and the forwarding nodes, the definition of them are in table 1.

Symbol definitions of the proposed algorithm are shown in table 2 .

Some parameters in the the proposed algorithm are defined as follows.

Definition 1. Two hop neighbors table TS(u): A table is composed by all neighbor nodes ID of node $u$ and all the corresponding two hop neighbor nodes ID. For example, table 3 is the two hop neighbors table of node 0 in Fig. 1 which is denoted TS $(0)$.

Definition 2. In-degree of two hop neighbor node $\mathrm{m} \operatorname{In}(\mathrm{m})$ : In two hop neighbors table, the number of neighbor nodes corresponding to the two hop neighbor node $\mathrm{m}$. For example in table $3, \operatorname{In}(10)=1, \operatorname{In}(9)=2, \operatorname{In}(12)=3$.

Definition 3. Out-degree of neighbor node $n$ Out(n): In two hop neighbors table, the number of two hop neighbor nodes corresponding to the neighbor node n. For example in table $3, \operatorname{Out}(1)=1, \operatorname{Out}(2)=2, \operatorname{Out}(7)=3, \operatorname{Out}(5)=4$.
TABLE I. DEFINITION OF NODES

\begin{tabular}{|l|l|}
\hline \multicolumn{1}{|c|}{ Symbol } & \multicolumn{1}{c|}{ Definition } \\
\hline Neighbor nodes & $\begin{array}{l}\text { The nodes in the transmission range } \\
\text { of node }\end{array}$ \\
\hline $\begin{array}{l}\text { Two hop neighbor } \\
\text { nodes }\end{array}$ & $\begin{array}{l}\text { The nodes in the transmission range } \\
\text { of neighbor node (Does not include } \\
\text { the node itself and its neighboring } \\
\text { nodes) }\end{array}$ \\
\hline Forwarding nodes & $\begin{array}{l}\text { The nodes which forward broadcast } \\
\text { packets after receiving the } \\
\text { broadcast packets }\end{array}$ \\
\hline
\end{tabular}

TABLE II. SYMBOL DEFINITION OF THE ALGORITHM

\begin{tabular}{|l|l|}
\hline \multicolumn{1}{|c|}{ Symbol } & \multicolumn{1}{c|}{ Definition } \\
\hline $\mathrm{N}(\mathrm{u})$ & The node u's set of neighbor nodes \\
\hline $\mathrm{N}_{2}(\mathrm{u})$ & $\begin{array}{l}\text { The node u's set of two hop neighbor } \\
\text { nodes }\end{array}$ \\
\hline $\operatorname{Re}(\mathrm{u})$ & The node u's set of the forwarding nodes \\
\hline
\end{tabular}

\section{B. Algorithm Description}

The core concept of 2-hop neighbors table based broadcasting algorithm is to choose fewer forwarding nodes covering maximum two hop neighbor nodes. The proposed algorithm is mainly include three processes: create two hop neighbors table, use two hop neighbors table to choose the forwarding nodes, complete broadcasting through forwarding nodes set. Forwarding nodes selection is the most important process in proposed algorithm.

In order to assure the connectedness of network, this paper assumes that there are not isolated nodes in the network and all nodes could receive messages when they are in the transmission range of a node. Each node does not have neighbor nodes information in the initial stage of network, they both creates two hop neighbors table TS(u) according to the rule. When TS(u) of every node has been created each node gets their own forwarding nodes set $\operatorname{Re}(\mathrm{u})$ according to the rule. When the node receives a broadcasting packet the node determine whether the packet is repeated, if it is a repeated packet the node discards it; otherwise the node determines whether itself is a forwarding node. If the node is a forwarding node it puts its ID and forwarding nodes set $\operatorname{Re}(\mathrm{u})$ into broadcasting packet, and forwards it out; otherwise it stops forwarding. When all nodes in the network stop forwarding, a broadcasting process has been completed.

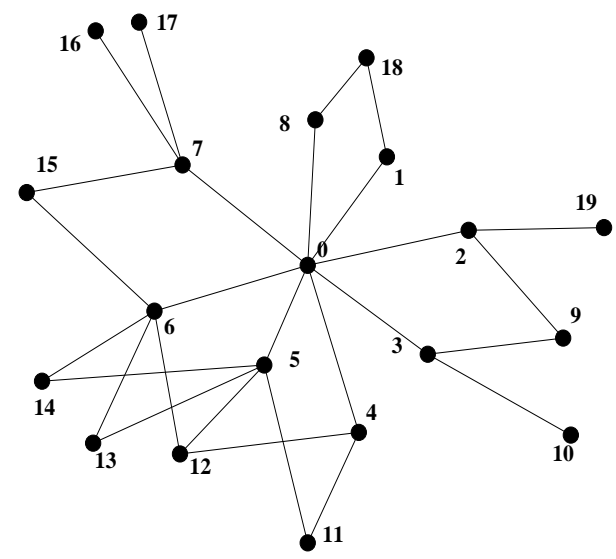

Figure 1. Topology of network 
TABLE III.

Two Hop NEIGHBors TABLE OF NODE 0

\begin{tabular}{|c|c|}
\hline ID of Neighbor Node & ID of Two Hop Neighbor Node \\
\hline 1 & 18 \\
\hline 2 & 9,19 \\
\hline 3 & 9,10 \\
\hline 4 & 11,12 \\
\hline 5 & $11,12,13,14$ \\
\hline 6 & $12,13,14,15$ \\
\hline 7 & $15,16,17$ \\
\hline 8 & 18 \\
\hline
\end{tabular}

\section{The Rule of Algorithm}

1) The rule of two hop neighbors table creation

S1 Each node in the network sends "Hello" packet to its neighbor nodes so that let its neighbor nodes know its presence.

S2 Node puts ID of neighbor nodes into N(u) after it receives the "Hello" packet of neighbor nodes, then sends packet with ID and $\mathrm{N}(\mathrm{u})$ of node itself to the neighbor nodes.

S3 Node puts $\mathrm{N}(\mathrm{u})$ of every node into corresponding two hop neighbor nodes set $\mathrm{N}_{2}(\mathrm{u})$ after it receives the "Hello" packet with $\mathrm{N}(\mathrm{u})$ of neighbor nodes and remove the neighbor nodes of itself in the corresponding $\mathrm{N}_{2}(\mathrm{u})$

S4 Node creates two hop neighbors table TS(u) according to $\mathrm{N}(\mathrm{u})$ and $\mathrm{N}_{2}(\mathrm{u})$ corresponding with each neighbor node.

2) The rule of forwarding nodes selection

S1 Node $u$ creates set $\operatorname{Re}_{1}(\mathrm{u})$ and $\operatorname{Re}_{2}(\mathrm{u})$, and initializes $\operatorname{Re}_{1}(\mathrm{u}), \operatorname{Re}_{2}(\mathrm{u})$ to empty set. Node u creates a new two hop neighbors table which is completely same with TS(u), denoted it $\mathrm{TS}_{0}(\mathrm{u})$.

$\mathrm{S} 2$ Node $\mathrm{u}$ finds out the two hop neighbor nodes from $\mathrm{TS}_{0}(\mathrm{u})$ which in-degree is equal to 1 , and put the corresponding neighbor nodes into $\operatorname{Re}_{1}(\mathrm{u})$.

S3 Node $u$ removes the neighbor nodes from $\mathrm{TS}_{0}(\mathrm{u})$ which are in $\operatorname{Re}_{1}(u)$, and the two hop neighbor nodes which are corresponding with the neighbor nodes in $\operatorname{Re}_{1}(\mathrm{u})$

S4 Node u judges whether it has two hop neighbor nodes in $\mathrm{TS}_{0}(\mathrm{u})$, go to $\mathrm{S} 5$ if it does not have. Otherwise node $\mathrm{u}$ finds out the neighbor node from $\mathrm{TS}_{0}(\mathrm{u})$ which has the maximal out-degree and adds into $\operatorname{Re}_{2}(u)$. (Node $u$ selects the node which has the smallest ID if some nodes have the same out-degree and their out-degrees are both the biggest.) Node $u$ removes the neighbor nodes and their corresponding two hop neighbor nodes from $\mathrm{TS}_{0}(\mathrm{u})$, then go to S4.

S5 Node $u$ let $\operatorname{Re}_{1}(\mathrm{u})$ plus $\operatorname{Re}_{2}(\mathrm{u})$ to get the forwarding nodes set $\operatorname{Re}(\mathrm{u})$. Node $\mathrm{u}$ delete $\mathrm{TS}_{0}(\mathrm{u})$ and the proposed algorithm ends up.

\section{SIMULATION}

This paper simulates 2NTB broadcasting algorithm in NS-2 (version 2.35) simulator, compares the proposed algorithm with Cluster broadcasting algorithm [12] and WSAB broadcasting algorithm [10]. This paper discusses the influence of three algorithms' performance in different number and different transmission range of nodes. The simulation environment is a rectangular area, all of nodes are distributed randomly in the rectangular area. Experimental parameters are showed in table 4, the result is the average of 100 times simulation.
TABLE IV. $\quad$ EXPERIMENTAL PARAMETER

\begin{tabular}{|l|l|}
\hline \multicolumn{1}{|c|}{ Parameter } & \multicolumn{1}{c|}{ Value } \\
\hline MAC Layer & IEEE 802.11 \\
\hline Propagation Model & two-ray ground \\
\hline Size of Square Area & $1000 \mathrm{~m} \times 1000 \mathrm{~m}$ \\
\hline Bandwidth & $2 \mathrm{M} \mathrm{bps}$ \\
\hline Packet Size & $256 \mathrm{byte}$ \\
\hline Maximum speed of nodes & $20 \mathrm{~m} / \mathrm{s}$ \\
\hline
\end{tabular}

\section{A. The influence of algorithm performance for different} node number

To analyze the influence of algorithm performance for different node number this paper fixes transmission range of node, and sets the transmission range of node to $250 \mathrm{~m}$. The number of nodes changes from 200 to 1000, and the simulation results are showed in Fig. 2, Fig. 3. Three algorithms' ratio of forwarding both decrease gradually with the number of nodes increasing. 2NTB algorithm's ratio of forwarding is always lower than Cluster algorithm. 2NTB algorithm's ratio of forwarding is lower than WSAB algorithm before the number of nodes increase to 1000; 2NTB algorithm's ratio of forwarding is equal to WSAB algorithm when the number of nodes is close to 1000. 2NTB algorithm's ratio of forwarding is not much different from Cluster algorithm and WSAB algorithm when the number of nodes is more than 600; however 2NTB algorithm's ratio of forwarding is lower than other two algorithms obviously when the number of nodes is less than 600. Three algorithms' transmission delays both decrease gradually with the number of nodes increasing, however 2NTB algorithm's transmission delay is always lower than Cluster algorithm and WSAB algorithm. The performance of $2 \mathrm{NTB}$ algorithm is better than Cluster algorithm and WSAB algorithm.

\section{B. The influence of algorithm performance for different transmission range}

This paper fixes number of nodes, and sets the number of nodes to 200. Transmission range of nodes changes from 150 to 500, the simulation result is showed in Fig. 4. Three algorithms' ratios of forwarding both reduce gradually with the transmission range of nodes increasing. 2NTB algorithm's ratio of forwarding is always lower than WSAB algorithm. 2NTB algorithm's ratio of forwarding is lower than Cluster algorithm obviously when the transmission range of nodes is smaller than $300 \mathrm{~m} ; 2 \mathrm{NTB}$ algorithm's ratio of forwarding is not much different from WSAB algorithm when the transmission range of nodes is bigger than $300 \mathrm{~m}$. This shows that the performance of 2NTB algorithm is better than Cluster algorithm obviously in the network with smaller transmission range. 


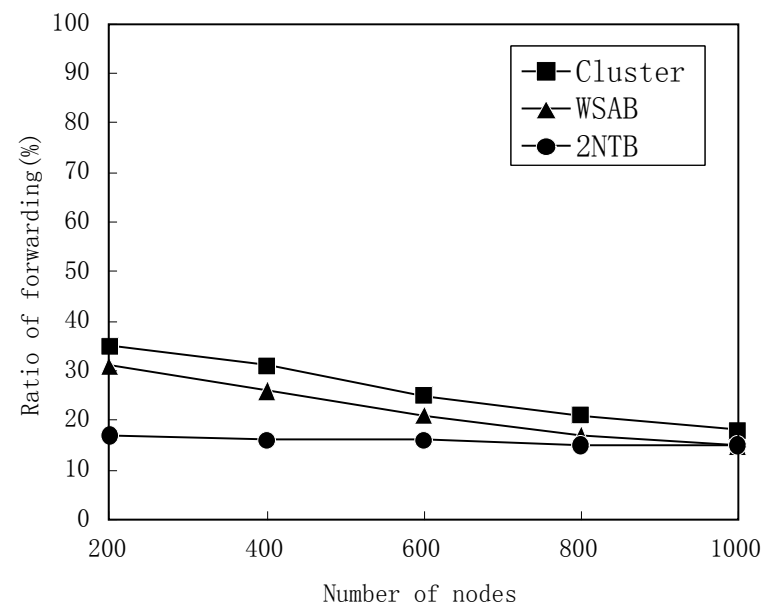

Figure 2. Ratio of forwarding versus number of nodes

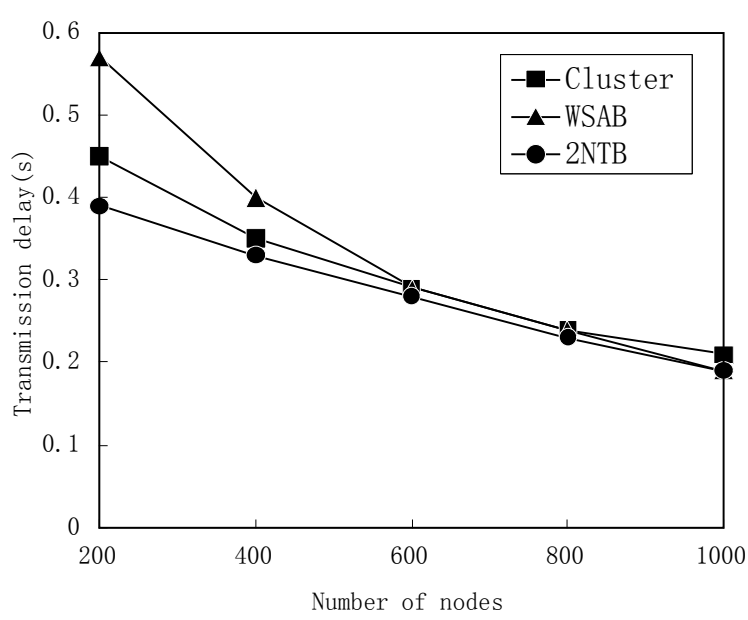

Figure 3. Transmission delay versus number of nodes

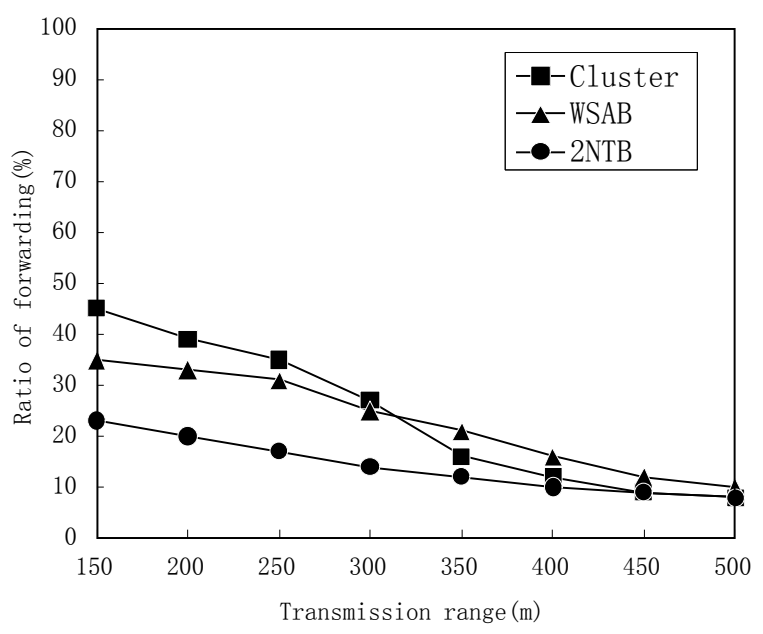

Figure 4. Ratio of forwarding versus transmission range

\section{CONCLUSIONS}

This paper proposes a 2-hop neighbors table based broadcasting algorithm. The proposed algorithm introduces two conceptions according to two hop neighbors table which are out-degree of neighbor nodes and in-degree of two hop neighbor nodes. Using outdegree and in-degree chooses forwarding nodes so that broadcasting is completed with less forwarding nodes. The simulation results show that the ratio of forwarding and transmission delay of the proposed algorithm are both better than other two broadcasting algorithms in different number of nodes and different transmission range. The proposed algorithm has better performance in the sparse network.

\section{ACKNOWLEDGMENT}

This research was financially supported by the natural science foundation of Liaoning Province (201102180).

\section{REFERENCES}

[1] Tseng Y C, Ni S Y, Chen Y S, et al. The broadcast storm problem in a mobile ad hoc network [J]. Wireless networks, 2002, 8(2-3): 153-167.J. Clerk Maxwell, A Treatise on Electricity and Magnetism, 3rd ed., vol. 2. Oxford: Clarendon, 1892, pp.68-73.

[2] Khan I A, Javaid A, Qian H L. Distance-based dynamically adjusted probabilistic forwarding for wireless mobile ad hoc networks[C]// IEEE 5th International Conference on Wireless and Optical Communications Networks, 2008: 1-6.

[3] Cai Y, Hua K A, Phillips A. Leveraging 1-hop neighborhood knowledge for efficient flooding in wireless ad hoc networks [C]. IEEE 24th International Conference on Computing and Communications, 2005: 347-354.

[4] Yassein M B, Nimer S F, Al-Dubai A Y. A new dynamic counterbased broadcasting scheme for Mobile Ad hoc Networks[J]. Simulation Modelling Practice and Theory, 2011, 19(1): 553-563.

[5] CHEN Wei-yu, SUN Wei-wei. A Survey on the Neighbor Based Broadcast Protocols in Ad Hoc Networks [J]. COMPUTER ENGINEERING \& SCIENCE, 2010,2(32): 15-19.

[6] Khabbazian M, Blake I F, Bhargava V K. Local Broadcas Algorithms in Wireless Ad Hoc Networks: Reducing the Number of Transmissions [J]. IEEE Transactions on Mobile Computing, 2012, 11(3): 402-413.

[7] Liu H, Jia X H, Wan P J, Liu X X, and Yao F. A distributed and efficient flooding scheme using 1-hop information in mobile Ad Hoc networks [J]. IEEE Transactions on Parallel and Distributed Systems, 2007, 18(5): 658-671.

[8] JiangHong Guo, ZhiChu Zhong. Efficient and Reliable Broadcast Protocol for Clustered Wireless Sensor Networks[C]// Proceedings - 2010 International Conference on Computational Intelligence and Security, CIS 2010: 488-492.

[9] Tian H, He Y, Zhang X, et al. An Approximation Algorithm for MCDS Construction in Ad Hoc Networks [J]. Future Wireless Networks and Information Systems. Springer Berlin Heidelberg, 2012: 65-71.

[10] LIU Juan-mei, WANG Xiao-ling. Symmetrical Area Broadcasting Algorithm in Ad Hoc Network [J]. Computer Engineering, 2011, 37(2).

[11] Wu J, Dai F. Broadcasting in ad hoc networks based on selfpruning $[\mathrm{J}]$.International Journal of Foundations of Computer Science, 2003, 14(02): 201-221.

[12] Foroozan F, Tepe K. A high performance cluster-based broadcasting algorithm for wireless ad hoc networks based on a novel gateway selection approach [C]// Proceedings of the 2nd ACM international workshop on Performance evaluation of wireless ad hoc, sensor, and ubiquitous networks, 2005: 65-70. 